\title{
Keine additiven Effekte der Kombination
}

Fragestellung: Ist die Kombination von einem Interferon mit Glatirameracetat in der Behandlung der schubförmigen Multiplen Sklerose (MS) wirksamer als die Behandlung mit den jeweiligen Einzelsubstanzen?

Hintergrund: Seit fast 20 Jahren werden MS-Patienten mit Interferonen und Glatirameracetat behandelt. Unklar ist jedoch bis heute, ob die Kombination dieser beiden Substanzen nicht doch einen additiven Effekt hinsichtlich der Wirksamkeit haben könnte, da deren Wirkmechanismen unterschiedlich sind. Die CombiRX (Combination Therapy in Patients With Relapsing-Remitting Multiple Sclerosis)-Studie wurde von den „National Institutes of Health “ (NIH) finanziert. Die Herstellerfirmen stellten nur die Medikation zur Verfügung. Die Studie begann bereits 2001.

Patienten und Methodik: In dieser über drei Jahre geführten Multicenterstudie $(\mathrm{n}=1.008)$ wurden Patienten mit schubförmiger MS-Verlaufsform in drei Studienarme eins zu eins zu zwei randomisiert, um die klini-

Lublin FD, Cofield SS, Cutter GR et al. for the CombiRX study group. Randomized study combining interferon and glatiramer acetate in multiple sclerosis. Ann Neurol 2013; DOI: 10.1002/ana.23863 sche Wirksamkeit von Interferon-beta la i.m. mit Glatirameracetat oder die Kombination beider Präparate zu vergleichen. Um ein möglichst aussagefähiges Ergebnis zu erhalten, wurden alle Patienten in einem Double-dummy-

Verfahren behandelt, das heißt alle Patienten mussten sich täglich subkutan und einmal wöchentlich intramuskulär behandeln, womit alle Patienten insgesamt acht Injektionen pro Woche erhielten. Eingeschlossen wurden Patienten mit schubförmiger MS im Alter zwischen 18 und 60 Jahren, die in der Lage waren mindestens 100 Meter mit oder ohne Gehhilfe zu gehen und mindestens zwei Schübe in den letzten drei Jahren erlitten hatten.

Ergebnisse: Die jährliche Schubrate während der drei Jahre andauernden Studie betrug 0,12 in der Kombinationsgruppe, 0,11 in der Glatirameracetat-Gruppe und 0,16 in der Interferon-Gruppe $(p=0,02)$. Der Prozentsatz der Patienten, die in den drei Studienjahren einen Schub erlitten, betrug in der Kombinationsgruppe $23 \%$, unter Glatirameracetat $23,1 \%$ und unter Interferonbeta la i.m. 26\%. Diese Unterschiede waren statistisch nicht signifikant. Auch hinsichtlich der Erkrankungsprogression ergaben sich keine signifikanten Unterschiede. Lediglich in einigen wenigen MRT-Parametern (Zahl der Kontrastmittel aufnehmenden Herde) gab es kleine, auch signifikante Unterschiede zugunsten der Kombinationstherapie. Neue oder unbekannte Nebenwirkungen traten nicht auf. Hinsichtlich des Nebenwirkungsspektrums zeigten sich ebenfalls keine additiven Effekte.

Schlussfolgerungen: Die Autoren schließen aus den Daten, dass die Kombination von Interferon-beta 1a i. m. mit Glatirameracetat $20 \mathrm{mg}$ s.c./d klinisch keinen Vorteil gegenüber der Einzelgabe der Substanzen hat und daher nicht empfohlen werden kann.

\section{-Kommentar von Volker Limmroth, Köln-Merheim}

\section{Interessant, aber zehn Jahre zu spät}

Diese Arbeit ist eigentlich spannend, kommt aber leider zehn Jahre zu spät. Nach den Zulassungen von Natalizumab, Fingolimod und der bevorstehenden Zulassung von BG-12, Alemtuzumab oder pegyliertem Interferon, das nur alle vier Wochen gespritzt werden muss, kommt natürlich niemand mehr auf die Idee, zwei Substanzen, die zum Teil täglich injiziert werden müssen, miteinander zu kombinieren. Leider hatte in den späten 1990er-Jahren kein pharmazeutisches Unternehmen Interesse an einer Kombination dieser Substanzen, sodass die Studie tatsächlich von einer staatlichen Stelle, den National Institutes of Health, bezahlt und organisiert werden musste. Die Studie hat mit über 1.000 Patienten eine beachtliche Größe und darf als valide gelten. Es ist auch die größte jemals prospektiv durchgeführte Vergleichsstudie zwischen einem Interferon und Glatirameracetat beziehungsweise einer aktiven Vergleichssubstanz. Die Studie hätten wir also gerne bereits im Jahr 2002 gehabt. Dennoch ist das Ergebnis pathophysiologisch interessant. Die Kombination aus Interferon und Glatirameracetat hat (von einigen wenigen sensiblen MRT-Parametern abgesehen) gar keine echten additiven Effekte. Dieses Ergebnis ist nicht einfach zu erklären, wenn man davon ausgeht, dass beide Substanzen unterschiedliche Wirkmechanismen haben. Ehrlicherweise muss aber auch eingestanden werden, dass zwar zur Wirkweise von Interferonen und Glatirameracetat eine Vielzahl von Theorien bestehen, die genauen Mechanismen aber bis heute nicht abschließend geklärt sind. Dagegen haben wir die Wirkmechanismen von Natalizumab und Fingolimod wesentlich besser verstanden. Möglicherweise ist also die Endstrecke von Interferonen und Glatirameracetat doch ähnlicher als bisher vermutet.

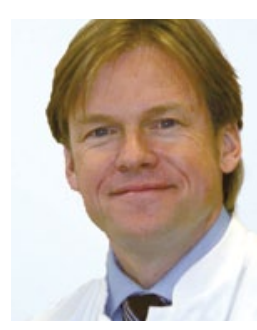

Prof. Dr. med. Volker Limmroth, Köln-Merheim

Chefarzt der Klinik für Neurologie und Palliativmedizin Köln-Merheim E-Mail: LimmrothV@kliniken-koeln.de 